04

\title{
Измерение электронной температуры плазмы фольговым рентгеновским спектрометром, установленным на токамаках ТУМАН-3М и Глобус-М2
}

\author{
(C) А.В. Воронин, ${ }^{1}$ В.Ю. Горяинов, ${ }^{1,2}$ В.В. Забродский, ${ }^{1}$ Е.В. Шерстнев, ${ }^{1}$ В.А. Корнев, ${ }^{1}$ П.Н. Аруев, ${ }^{1}$ \\ Г.С. Курскиев, ${ }^{1}$ Н.А. Жубр, ${ }^{1}$ А.С. Тукачинский ${ }^{1}$ \\ ${ }^{1}$ Физико-технический институт им. А.Ф. Иофффе РАН, \\ 195251 Санкт-Петербург, Россия \\ ${ }^{2}$ Санкт-Петербургский политехнический университет Петра Великого, \\ 195251 Санкт-Петербург, Россия \\ e-mail: vgoryainov@mail.ioffe.ru
}

Поступило в Редакцию 18 июня 2021 г.

В окончательной редакции 12 августа 2021 г.

Принято к публикации 24 августа 2021 г.

Представлено техническое решение фольгового спектрометра, установленного на токамаках Глобус-М2 и ТУМАН-3М для измерения электронной температуры плазмы. Проведены измерения зависимости температуры плазмы от времени в центральной области токамаков. Применение интегрированных фотоприемников и уникальных бериллиевых фольг с толщинами $14-80 \mu \mathrm{m}$ позволило увеличить чувствительность спектрометра. Важным качеством используемых фольг являлись повышенные значения прочности, пластичности, однородности, отсутствие поверхностных и внутренних дефектов. Совместное использование спектрометра с диагностикой томсоновского рассеяния позволило проводить регулярные измерения температуры в токамаке Глобус-М2 с высокими пространственным и временным разрешениями. Проведена оценка влияния примесей на измерение электронной температуры плазмы.

Ключевые слова: фольговая спектрометрия, токамак, плазма, континуум, тормозное излучение, электронная температура, мягкое рентгеновское излучение, кремниевый фотодиод.

DOI: 10.21883/JTF.2021.12.51758.188-21

\section{Введение}

Диагностические методы, основанные на измерении спектра и интенсивности рентгеновского излучения, успешно используются в исследовании плазмы. В сочетании с другими методами исследований рентгеновская диагностика весьма полезна для изучения различных процессов, происходящих в плазме токамака. Например, она позволяет исследовать внутренние МГД-колебания плазмы и определять расположение поверхностей с рациональным значением запаса устойчивости $[1,2]$, оценивать потери электронов за счет электронно-циклотронной эмиссии ЕСЕ [3] и убегающих электронов [4], ELM-события [5,6], отслеживать L-Hпереходы [7] и многое другое [8-16]. Такие устройства широко применяются на токамаках JET [17], MAST [8], DIII-D [18], Asdex-Upgrade [19], PDX [20], NSTX [2123], EAST [5], MST [24], TCV [25,26], COMPASS [27,28]. В рентгеновских устройствах измерение производится без вмешательства в ход изучаемого процесса.

Аппаратура для регистрации температуры является одной из самых востребованных при изучении поведения лабораторной плазмы. В настоящее время электронную температуру определяют по величине проводимости плазмы, собственному излучению интенсивности линейчатого и сплошного спектра плазмы, а также электронно-циклотронной частоты и ее гармоник [29-31].

В настоящей работе представлено техническое решение фольгового рентгеновского многоканального спектрометра, установленного на токамаках Глобус-М2 и ТУМАН-3М для определения электронной температуры плазмы на оси плазменного шнура с высоким временным разрешением.

На сферическом токамаке Глобус-М2 (малый радиус $a=0.24 \mathrm{~m}$, большой радиус $R=0.36 \mathrm{~m}$, магнитное поле на оси тора $B_{T}=0.5-0.9 \mathrm{~T}$, плазменный ток $\left.I_{P}=150-400 \mathrm{kA}\right)[32]$ для измерения электронной компоненты плазмы применяли две взаимодополняющие диагностики - томсоновское рассеяние луча лазера во время его взаимодействия с плазмой [33] и фольговую спектрометрию мягкого рентгеновского излучения. С помощью лазера с частотой импульсов $300 \mathrm{~Hz}$ проводилась регистрация температуры в 10 пространственных областях плазменного шнура в течение разряда токамака. Высокая стоимость и ограниченный срок работы лазера не позволяли постоянно использовать эту диагностику. Для разработанного фольгового спектрометра, помимо пассивности измерения, характерны невысокая цена, небольшие габариты, простая эксплуатация по сравнению с томсоновской диагностикой. 
Фольговый спектрометр обеспечивал непрерывное измерение температуры с частотой дискретизации $\sim 50 \mathrm{kHz}$ и применялся в течение всей экспериментальной кампании. При этом реальная частота спектрометра в составе токамака Глобус-М составляла $30 \mathrm{kHz}$. Она ограничивалась полосой пропускания оптической развязки, встроенной в систему сбора данных токамака. Основным недостатком этой диагностики являлась большая погрешность вычисления электронной температуры. Однако она была существенно дешевле диагностики томсоновского рассеяния. Для проведения измерений электронной температуры водородной/дейтериевой плазмы необходимо предположение о максвелловском распределении электронов по энергиям. Также требуется чистота плазмы от примесей и значение электронной температуры $T_{e}>100 \mathrm{eV}$.

На токамаке ТУМАН-3М $(a=0.22 \mathrm{~m}, R=0.53 \mathrm{~m}$, $\left.B_{T}=1.0 \mathrm{~T}, I_{P}=190 \mathrm{kA}\right)$ [34] измерение температуры электронов проводилось с помощью двух фольговых спектрометров. Они отличались друг от друга расположением на токамаке, чувствительностью, быстродействием и конструкцией. Датчики и фольги основного спектрометра (всего 9 хорд), установленного ранее на токамак, находились в инертном газе и соединялись с вакуумной камерой через бериллиевую фольгу толщиной $50 \mu \mathrm{m}$. Датчики с предусилителями и тонкие фольги другого спектрометра, разработанного сейчас, располагались внутри вакуумной камеры токамака и имели более высокое временное разрешение, чем датчики основного спектрометра. Электронная температура, измеренная разработанным спектрометром, сравнивалась с измерениями температуры основного спектрометра.

\section{1. Фольговый спектрометр}

В свободной от примесей водородной/дейтериевой плазме при электронной температуре более $100 \mathrm{eV}$ тормозное рентгеновское непрерывное излучение, возникающее из-за ускорения электронов в кулоновском поле ионов, играет главную роль по сравнению с рекомбинационным, линейчатым и циклотронным излучениями [35]. Спектральную плотность мощности тормозного излучения в единице объема плазмы $d V$ с учетом максвелловского распределения электронов по энергиям можно описать формулой $[13,36]$ :

$$
\frac{d P_{f f}}{d v \cdot d V} \approx 10^{-28} \cdot Z_{\mathrm{eff}}^{2} \cdot\left(g_{f f}\right) \cdot n_{i} \cdot n_{e} \cdot \sqrt{\frac{\chi_{H}}{T_{e}}} \cdot \exp \left(-\frac{h v}{T_{e}}\right),
$$

где $\chi_{H}-$ потенциал ионизации водорода, $T_{e}-$ температура электронов, $[\mathrm{eV}], n_{i}$ и $n_{e}-$ плотности ионов и электронов соответственно, $\left[\mathrm{cm}^{-3}\right], h-$ постоянная Планка, $v$ - частота фотонов, $Z_{\mathrm{eff}}$ - эффективный заряд плазмы, $\left\langle g_{f f}\right\rangle-$ усредненный по энергиям Гаунт-фактор для свободно-свободных переходов, который может быть вычислен в соответствии с [37]. Если энергия фотона $\varepsilon=h \cdot v$, то интенсивность тормозного излучения можно представить в виде

$$
I_{f f} \propto f\left(T_{e}, n_{e}, Z_{\mathrm{eff}}\right) \cdot \exp \left(-\frac{\varepsilon}{T_{e}}\right) .
$$

Здесь $f-$ функция, зависящая от электронной температуры, плотности, эффективного заряда и фактора Гаунта, энергия фотона $\varepsilon$ выражена в eV. Если это излучение попадает на диод с фольгой определенной толщины, то сигнал с детектора можно представить в виде

$$
i(t) \propto f\left(T_{e}, n_{e}, Z_{\mathrm{eff}}\right) \cdot \int_{0}^{\infty} F^{F P U}(\varepsilon) \cdot F^{f o i l}(\varepsilon) \cdot \exp \left(-\frac{\varepsilon}{T_{e}}\right) d \varepsilon
$$

где $F^{F P U}$ и $F^{\text {foil }}$ - спектральные характеристики диода и фильтра соответственно.

При использовании двух детекторов с фольгами разной толщины, принимающих рентгеновское излучение из одной и той же области, можно определить температуру электронов из отношения

$$
\frac{i_{1}}{i_{2}}=R\left(T_{e}\right)=A \cdot \frac{\int_{0}^{\infty} F^{F P U}(\varepsilon) \cdot F_{1}^{f o i l}(\varepsilon) \exp \left(-\frac{\varepsilon}{T_{e}}\right) d \varepsilon}{\int_{0}^{\infty} F^{F P U}(\varepsilon) \cdot F_{2}^{f o i l}(\varepsilon) \exp \left(-\frac{\varepsilon}{T_{e}}\right) d \varepsilon},
$$

где $A-$ коэффициент, учитывающий различие каналов регистрации датчиков, который определялся в результате калибровки прибора по эталонному источнику излучения. Индексы 1 и 2 соответствовали фольгам детекторов различной толщины. Интегрирование по энергии фотона производили по области чувствительности детектора $10 \mathrm{eV}-50 \mathrm{keV}$.

Зная функцию $R\left(T_{e}\right)$ и амплитуды сигналов с детекторов, можно получить значение температуры для каждого момента времени. Изменяя толщины фольг, можно изменять чувствительность прибора к разным диапазонам температур. Использование нескольких каналов (более двух) с различными толщинами фольг позволяло выбрать рабочую область спектра без наличия излучения примесей. С этой целью в настоящей работе представлены четырехканальный и трехканальный спектрометры для токамаков Глобус-М2 и ТУМАН-3М соответственно, которые позволяли проводить измерение температуры плазмы в центральной области плазменного шнура с временным разрешением $\sim 20 \mu$ s. Результаты измерений альтернативных диагностик обеспечивали дополнительную калибровку и сверку настоящих измерений.

Разработанный спектрометр состоял из детекторов, бериллиевых фольг, коллиматоров и стабилизированного источника питания. Внешний вид фланца, оснащенного детекторами, фильтрами и коллиматорами, а также схема спектрометра представлены на рис. 1. Все элементы прибора были смонтированы на фланце ДУ80 на стороне, обращенной в вакуумную камеру токамака. 

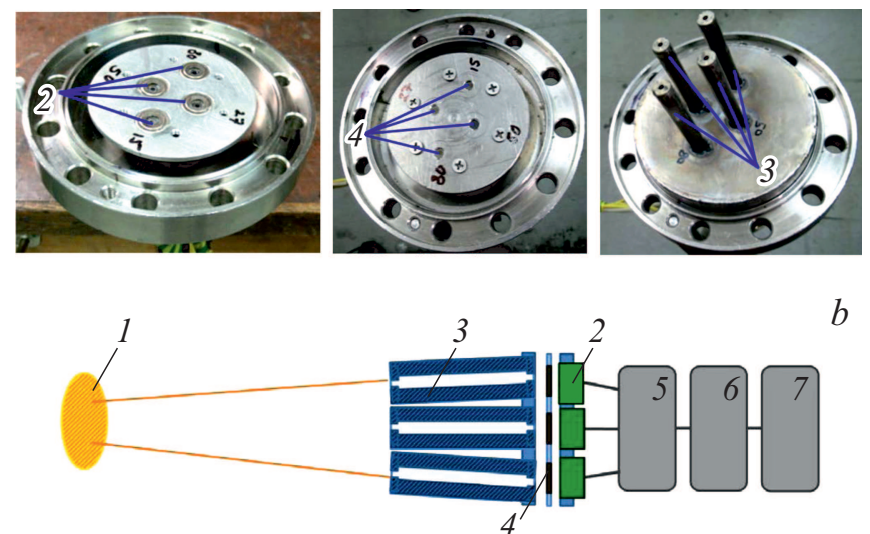

Pис. 1. $a-$ элементы четырехканального спектрометра, установленные на вакуумном фланце, слева направо: детекторы, фольги и коллиматоры; $b-$ схема фольгового спектрометра: 1 - плазма, 2 - фотодиоды с интегрированными усилителями, 3 - коллиматоры, 4 - бериллиевые фольги, 5 - стабилизированный источник питания, 6 - аналоговоцифровой преобразователь, 7 - компьютер.

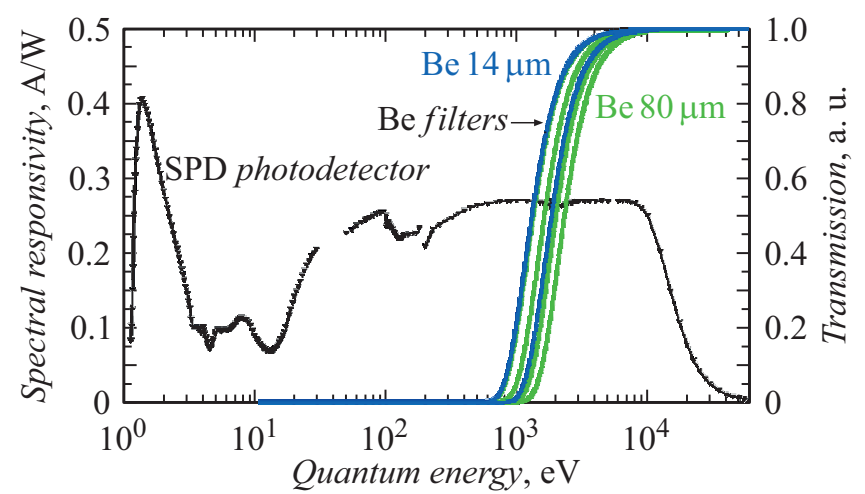

Рис. 2. Спектральная характеристика SPD фотодиода и пропускание бериллиевых фольг различной толщины.

Коллиматоры длиной $60 \mathrm{~mm}$ и диаметром входного отверстия $3 \mathrm{~mm}$ собирали излучение плазмы в экваториальной области токамака вдоль линии наблюдения с малым телесным углом.

Юстировка коллиматоров проводилась в видимой области спектра на стенде с помощью светодиодов, установленных вместо детекторов и фольг. На экране, расположенном на расстоянии, соответствующему расстоянию от детекторов до центра плазменного шнура (970 mm для Глобус-М2 и $580 \mathrm{~mm}$ для ТУМАН-3М), формировались изображения лучей от светодиодов в области их пересечения с экраном. Совмещение изображений производилось механическим изгибанием коллиматоров. Диаметр изображения пятна составлял $\sim 20 \mathrm{~mm}$.

Детекторы были изготовлены компанией $3 \mathrm{AO}$ „Техноэксан“ специально для этого спектрометра [38]. В применяемых детекторах SPD (silicon precision detector) $[39,40]$ был сформирован достаточно тонкий „мертвый слой“,
Основные параметры детектора

\begin{tabular}{l|c}
\hline \multicolumn{1}{c|}{ Усиление } & $10^{7} \mathrm{~V} / \mathrm{A}$ \\
\hline Напряжение шума & $<10 \mathrm{mV}$ \\
Диаметр активной области & $3.2 \mathrm{~mm}$ \\
Отношение сигнал/шум & $\sim 100$ \\
Фронт нарастания & $<20 \mu \mathrm{s}$
\end{tabular}

что позволяло получать большой сигнал на выходе. К каждому детектору был подсоединен трансимпендансный усилитель. Главная особенность разработанных детекторов состояла в их высокой чувствительности и временном разрешении, что позволило регистрировать быстрые процессы, происходящие в плазме токамака. Спектральная характеристика $F^{F P U}(\varepsilon)$ детекторов представлена на рис. 2 [40]. В таблице приведены основные электрические и динамические характеристики детектора.

Питание и сигналы с детекторов подключались через вакуумные разъемы, расположенные на фланце. Источник питания на выходе обеспечивал напряжение $\pm 5 \mathrm{~V}$.

Бериллиевые фольги были изготовлены в Институте машиностроения, материалов и транспорта при Политехническом университете Петра Великого [41,42] с помощью многопроходной горячей прокатки. Важным качеством этих фольг (с минимально возможной толщиной $5 \mu \mathrm{m}$ ) являлись повышенные значения прочности, пластичности, однородности, отсутствие поверхностных и внутренних дефектов. Толщины фольг, установленных на фотодетекторах спектрометра в токамаке Глобус-М2, составляли 15, 27, 50 и $80 \mu \mathrm{m}$. На токамаке ТУМАН-3М - 27, 14 и $40 \mu \mathrm{m}$. Спектральные характеристики фольг $F^{\text {foil }}$ рассчитывали с использованием базы данных [43]. Они представлены на рис. 2 справа.

Калибровка каналов спектрометра проводилась с помощью излучения инфракрасного светодиода на длине

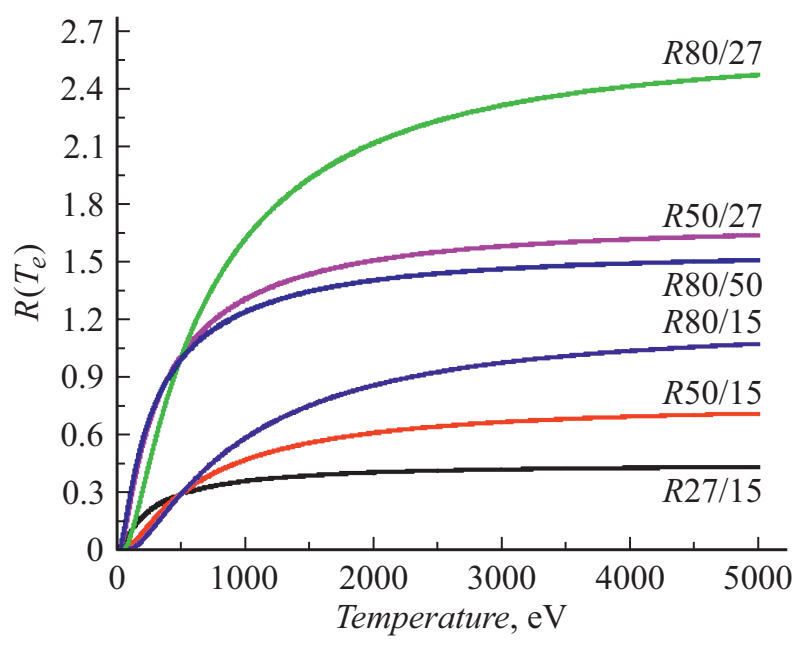

Рис. 3. Зависимости $R\left(T_{e}\right)$ для различных толщин $(15,27,50$, $80 \mu \mathrm{m})$ фольг, применяемых на токамаке Глобус-М2 с учетом калибровочного коэффициента $A$. 

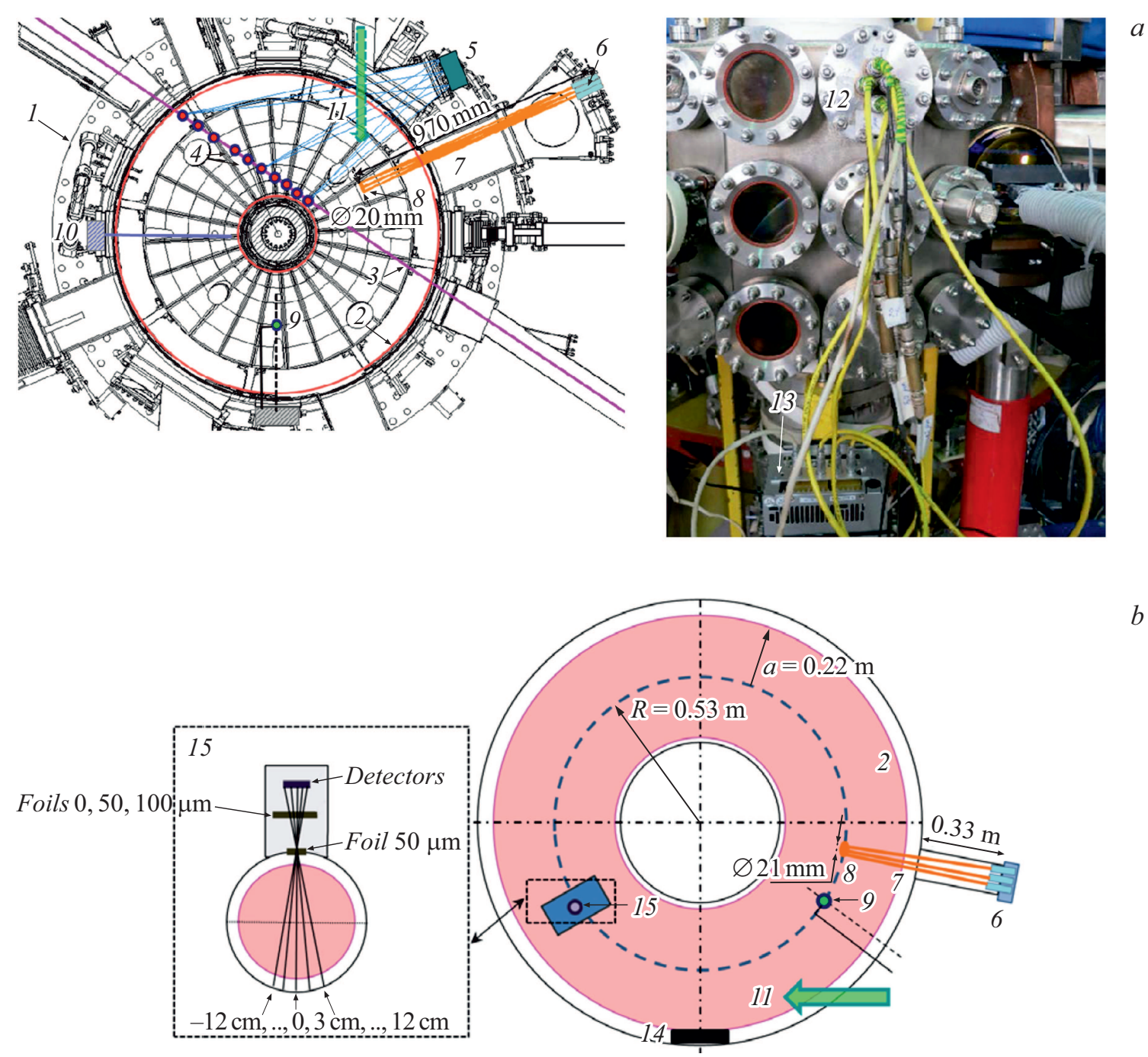

$b$

Pис. 4. $a-$ расположение диагностик и четырехканального спектрометра, установленных на токамаке Глобус-М2: $1-$ сечение камеры в экваториальной плоскости, 2 - внешняя граница плазмы, 3 - зондирующий лазерный луч, 4 - области измерения температуры лазером, 5 - объектив, 6-8- коллиматоры, границы сбора излучения и область измерения температуры спектрометра, 9 - области измерения среднехордовой плотности СВЧ-интерферометром, $10-$ фотоприемники для измерения излучения линии CIII, 11 - расположение и направление нейтральной инжекции NBI, 12,13 - фланец и источник питания спектрометра; $b-$ схема экваториального сечения токамака ТУМАН-3М: $14-$ лимитер, 15 - расположение и схема основного спектрометра.

волны $1050 \mathrm{~nm}$. Бериллиевые фольги во время калибровки отсутствовали. Оправка со светодиодом по очереди надевалась на один из четырех коллиматоров. Проводилось измерение напряжения на выходе детектора каждого канала. С учетом этих измерений определялся коэффициент $A$, необходимый для расчета зависимости отношения сигналов датчиков $R\left(T_{e}\right)$ от температуры. Результаты вычислений для токамака Глобус-М2 представлены на рис. 3. Видно, что наибольшая чувствительность $R\left(T_{e}\right)$ к температуре проявлялась тогда, когда выбирались пары фольг с наименьшей и наибольшей толщинами, но так, чтобы второй сигнал отличался от шума.

Процедура определения зависимости температуры от времени $T_{e}(t)$ состояла в сопоставлении расчетной функции отношения сигналов от температуры $R\left(T_{e}\right) \mathrm{c}$ экспериментальной зависимостью отношения сигналов от времени $R(t)$. Эта процедура выполнялась с помощью алгоритма, встроенного в программу Combiscope, разработанную для сбора и обработки данных токамака Глобус-М2. Аналогичная программа использовалась и на токамаке ТУМАН-3М.

Расположение диагностик и четырехканального спектрометра, установленных на токамаке Глобус-М2, представлено на рис. $4, a$.

Четырехканальный спектрометр располагался на патрубке выше экваториальной плоскости токамака, поэтому коллиматоры были наклонены в сторону экватора на угол $8^{\circ} 41^{\prime}$ для сбора излучения плазмы из центральной области вакуумной камеры. Также коллиматоры были 

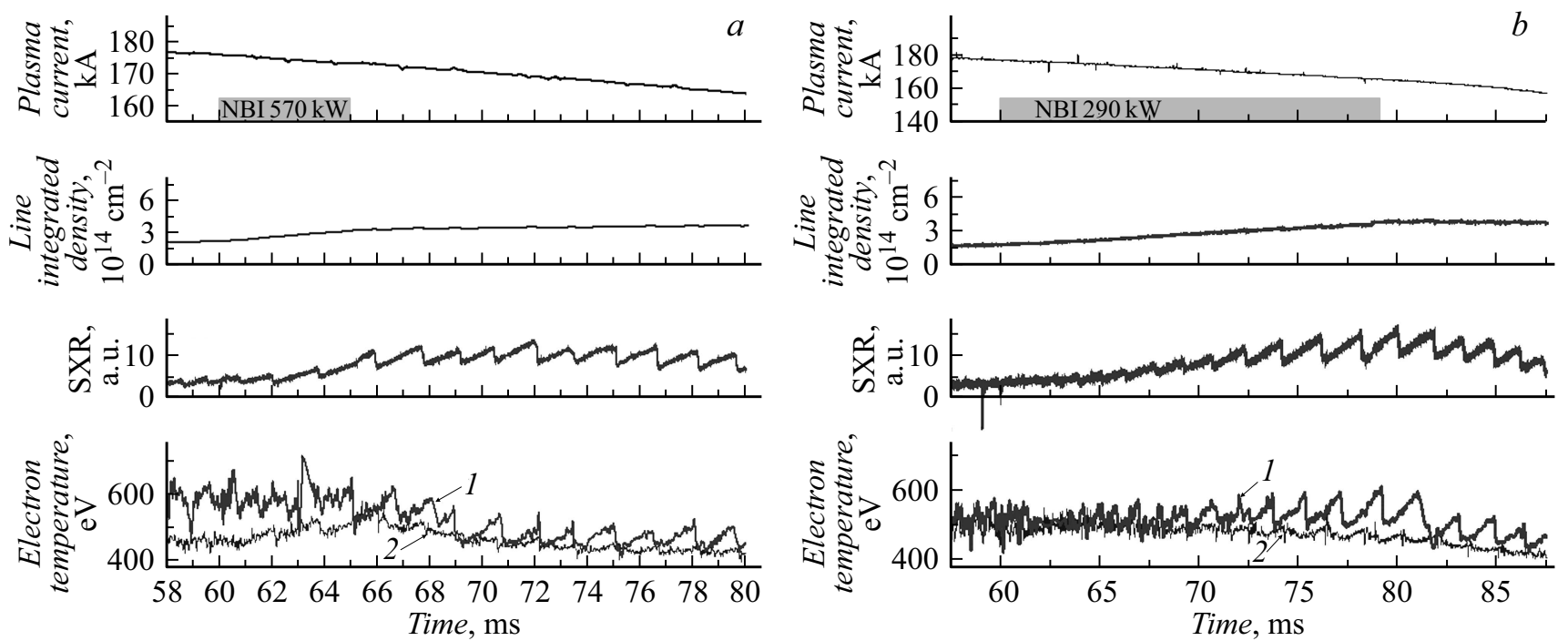

Рис. 5. Эволюции параметров разрядов \# 19022017 (a) и \# 19022014 (b) в токамаке ТУМАН-3М. 1 - температура электронной компоненты плазмы, полученная спектрометром с фольгами толщиной 14 и $27 \mu \mathrm{m}, 2$ - то же с фольгами толщиной 100 и $150 \mu \mathrm{m}$.

наклонены относительно друг друга для сбора излучения из одной области диаметром $20 \mathrm{~mm}$ на расстоянии $970 \mathrm{~mm}$ от детекторов (позиция 8 на рис. $4, a$ ).

С помощью диагностики томсоновского рассеяния проводилось измерение температуры в 10 пространственных областях плазменного шнура (позиция 4 на рис. 4,a) до 35 раз в течение разряда токамака. Длительность зондирующего лазерного импульса составляла $\sim 10-50 \mathrm{~ns}$, интервал между импульсами $\sim 2.5-3 \mathrm{~ms}$. Лазерный пучок проходил в экваториальной плоскости вблизи столба, система сбора рассеянного излучения также располагалась в экваториальной плоскости.

Схема экваториального сечения токамака ТУМАН-3М с диагностиками представлена на рис. $4, b$. Трехканальный разработанный спектрометр располагался в экваториальной плоскости. Крупным планом показана схема основной рентгеновской диагностики (позиция 15), с измерениями температуры которой производилось сравнение результатов, полученных трехканальным спектрометром.

\section{2. Результаты}

На рис. 5 представлена эволюция параметров разрядов дейтериевой плазмы на токамаке ТУМАН-3М с нейтральной инжекцией водорода различной длительности и мощности пучка. Цифрой 1 обозначена электронная температура, измеренная в радиальном направлении тора с помощью разработанного трехканального спектрометра с толщинами фольг 14 и $27 \mu \mathrm{m}$. Линия $2-$ температура, полученная основным спектрометром с толщинами фольг 100 и $150 \mu \mathrm{m}$. Видно, что во время инжекции пучка измеренные температуры совпадали, когда среднехордовая плотность плазмы превышала $3 \cdot 10^{14} \mathrm{~cm}^{-2}$. После завершения инжекции на обеих диагностиках наблюдалось уменьшение температуры. Величины, полученные с помощью пар фольг толщиной 40, 27 и 40, $14 \mu \mathrm{m}$, на трехканальном спектрометре оказывались заниженными, что могло быть связано с влиянием примесей нержавеющей стали [12], из которой состоял лимитер токамака. Видно, что трехканальный фольговый спектрометр обеспечивал измерение температуры с более высоким разрешением во времени по сравнению с разрешением, полученным с помощью основного спектрометра.

Пример возможностей фольгового спектрометра на токамаке Глобус-М2 продемонстрирован на рис. 6, где представлены процессы различных временных масштабов.

На рис. 6, а представлены параметры разряда с пилообразными колебаниями. Измерение температуры электронов в плазме проводилось одновременно с помощью фольгового спектрометра и диагностики томсоновского рассеяния. Максимальная температура радиального профиля, измеренного диагностикой томсоновского рассеяния, была на центральной хорде, наиболее близкой в полоидальной проекции к области измерения спектрометра. В обоих случаях обе диагностики фиксировали колебания температуры на оси плазменного шнура с характерной частотой $\sim 300 \mathrm{~Hz}$. Однако фольговый спектрометр обеспечивал измерение температуры с более высоким разрешением во времени по сравнению с разрешением, измеренным диагностикой томсоновского рассеяния. На рис. $6, b$ представлены параметры омического разряда, обусловленные возбуждением моды типа „снейк“. Видно, что фольговый спектрометр регистрировал колебания электронной температуры с характерной частотой $\sim 30 \mathrm{kHz}$.

Несоответствие результатов измерений электронной температуры, полученными спектрометром с различны- 

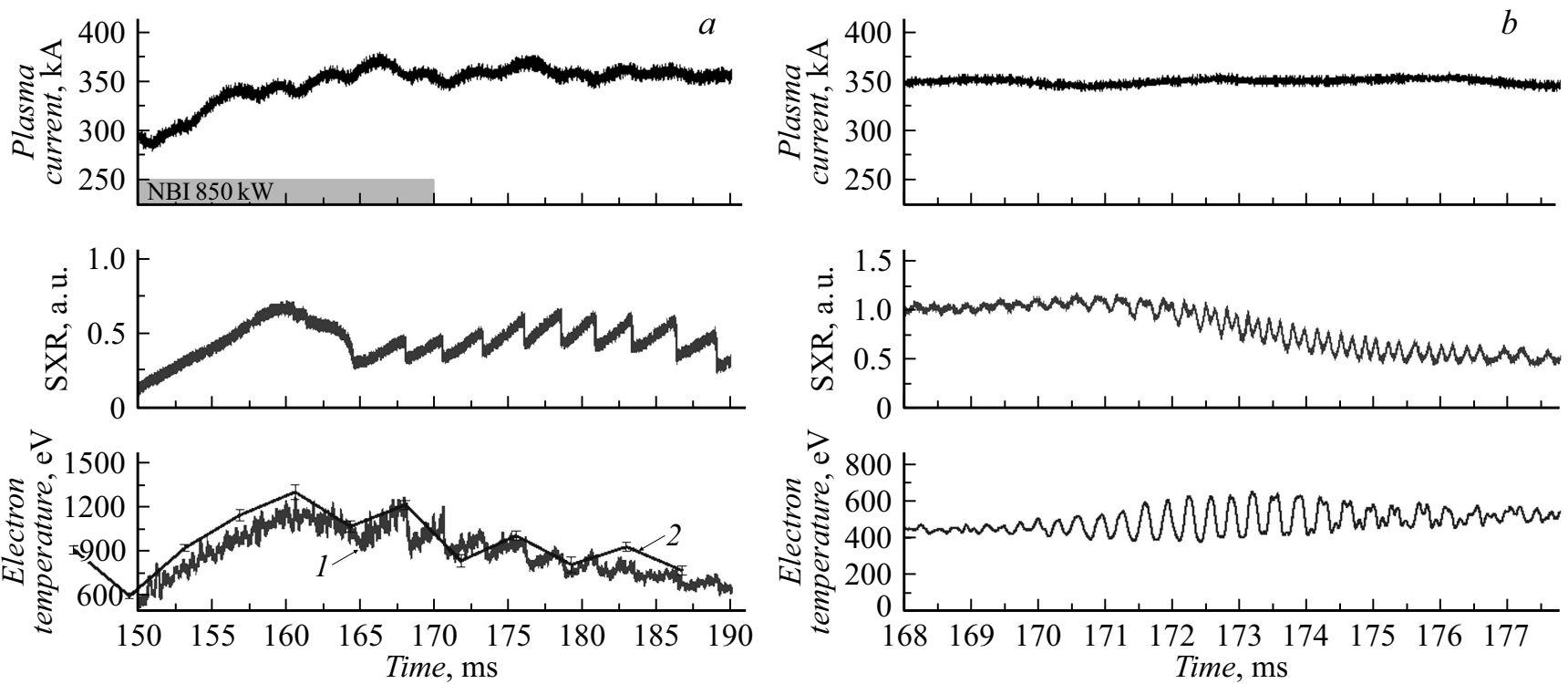

Рис. 6. Эволюции параметров плазменных разрядов в токамаке Глобус-М2. $a$ - разряд с пилообразными колебаниями, \# 38835 ; $b-$ разряд с возбуждением моды типа „снейк“, \# 38838. Температура электронной компоненты плазмы, полученная с помощью 1 - фольгового спектрометра, 2 - диагностики томсоновского рассеяния.

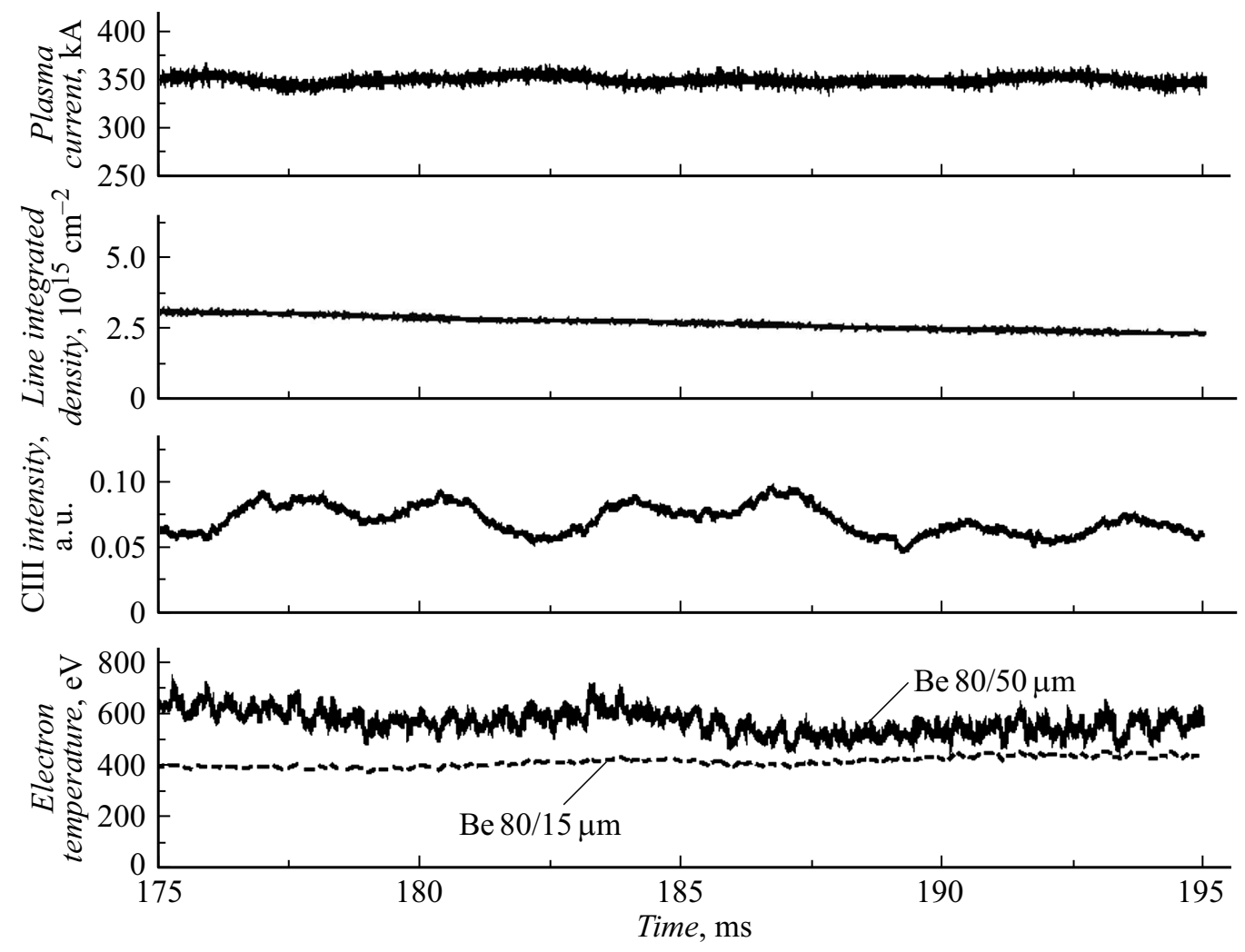

Рис. 7. Параметры разряда \# 38837 токамака Глобус-М2.

ми толщинами фольг, диагностике томсоновского рассеяния, могло быть вызвано влиянием излучения примесей углерода, так как первая стенка токамака Глобус-М2 была покрыта графитовыми тайлами. На рис. 7 представлены параметры омического разряда с умеренным количеством углерода в плазме. На нижнем графике показаны значения электронной температуры, измеренные спектрометром с парами бериллиевых фольг различной толщины. Приборная погрешность в измерении не превышала 20\%. Видно, что с увеличением тол- 
щин фольг измеренная температура заметно возросла. Можно предположить, что влияние излучения углерода уменьшалось по мере увеличения толщины фольг, а измеренная температура приближалась к истинной.

\section{Заключение}

Разработан, изготовлен и апробирован фольговый спектрометр для измерения температуры электронов плазмы в токамаках Глобус-М2 и ТУМАН-3М. Применение фотоприемников, интегрированных с усилителями, и уникальных тонких бериллиевых фольг позволило увеличить чувствительность и быстродействие спектрометра. Подготовлен алгоритм вычисления зависимости температуры от времени по измеренным сигналам мягкого рентгеновского излучения. Проведены измерения зависимости электронной температуры плазмы от времени в центральной области токамаков. Измеренные температуры в основном согласуются с температурами, полученными с помощью альтернативных диагностик. Совместное использование фольгового спектрометра с диагностикой томсоновского рассеяния позволило проводить регулярные измерения температуры в токамаке Глобус-М2 с высокими пространственным и временным разрешениями. Сравнение результатов на токамаке ТУМАН-3М показало применимость метода для бериллиевых фольг толщиной менее $100 \mu \mathrm{m}$. Проведена оценка влияния примесей на измерение электронной температуры плазмы фольговым спектрометром.

\section{Финансирование работы}

Разработка фольгового спектрометра была поддержана в рамках государственного задания ФТИ им. А.Ф. Иоффе 0040-2019-0023. Эксперименты проведены на УНУ „Сферический токамак Глобус-М“, входящей в состав ФЦКП „Материаловедение и диагностика в передовых технологиях“ (уникальный идентификатор проекта RFMEFI62119Х0021), а также на УНУ „ТУМАН-3М“. Работа поддержана Министерством науки и высшего образования Российской Федерации в рамках государственного задания в сфере науки по проекту № 07842020-0020.

\section{Конфликт интересов}

Авторы заявляют, что у них нет конфликта интересов.

\section{Список литературы}

[1] L. Xu, L. Hu, K. Chen, E. Li, F. Wang, M. Xu, Y. Duan, T. Shi, J. Zhang, R. Zhou, Y. Chen. Phys. Plasmas, 19, 122504 (2012).

[2] S. Mirnov. Physical Processes in Tokamak Plasma (Atomizdat, Moscow,1983), p. 116

[3] H. Lu, J. Luo, F. Zhong, X. Zha, L. Hu. Eur. Phys. J. D, 66, 213 (2012).
[4] А.С. Тукачинский, Л.Г. Аскинази, И.М. Балаченков, А.А. Белокуров, Д.Б. Гин, Н.А. Жубр, В.А. Корнев, С.В. Лебедев, Е.М. Хилькевич, И.Н. Чугунов, А.Е. Шевелев. Письма в ЖТФ, 42 (24), 72 (2016). [A. Tukachinsky, L. Askinazi, I. Balachenkov, A. Belokurov, D. Gin, N. Zhubr, V. Kornev, S. Lebedev, E. Khil'kevich, I. Chugunov, A. Shevelev. Tech. Phys. Lett., 42 (12), 1167 (2016).]

[5] K. Chen, L. Xu, L. Hu, Y. Duan, X. Li, Y. Yuan, S. Mao, X. Sheng, J. Zhao. Rev. Sci. Instrum., 87, 063504 (2016).

[6] Y. Li, G. Xu, K. Tritz, X. Lin, H. Liu, Y. Chen, S. Li, F. Yang, Z. Wu, L. Wang, H. Lan, X. Li, W. Zhang, G. Hu. Fusion Eng. Des., 137, 414 (2018).

[7] L. Askinazi, V. Kornev, S. Krikunov, L. Krupnik, S. Lebedev, A. Smirnov, M. Tendler, A. Tukachinsky, M. Vildjunas, N. Zhubr. J. Phys.: Conf. Ser. 123, 012010 (2008).

[8] M. Cecconello, O. Jones, L. Garzotti, K. McClements, M. Carr, S. Henderson, S. Sharapov, I. Klimek and the MAST Team. Nucl. Fusion. 55, 032002 (2015).

[9] T. Onchi, R. Ikezoe, K. Oki, A. Sanpei, H. Himura, S. Masamune. Rev. Sci. Instrum., 81, 073502 (2010).

[10] L. Askinazi, M. Vild'zhunas, V. Golant, V. Kornev, S. Krikunov, S. Lebedev, G. Razdobarin, V. Rozhdestvensky, E. Shevkin, A. Tukachinsky, S. Tsaun, N. Zhubr. 29th EPS Conference on Plasma Phys. and Contr. Fusion, Montreux, 17-21 June 2002 ECA, vol. 26B, P-2.070 (2002).

[11] M. Kantor, A. Altukhov, V. Belik, L. Esipov, D. Kouprienko L. Shmaenok, V. Yermolayev. 30th EPS Conference on Contr. Fusion and Plasma Phys., St. Petersburg, 7-11 July 2003 $E C A$, vol. 27, A P-2.60 (2003).

[12] S. von Goeler, W. Stodiek, H. Eubank, H. Fishman, S. Grebenshchikov, E. Hinnov. Nucl. Fus., 15 (2), 301 (1975).

[13] Huddlestone R and Leonard S. Plasma Diagnostic Techniques (Academic Press, NY.-London, 1965), p. 304.

[14] V. Andreev, I. Bragin. Plasma Diagnostics with Conductivity Probes (St.-Petersburg: SUAI 2000), p. 4.

[15] K. Nishimura, A. Sanpei, H. Tanaka, G. Ishii, R. Kodera, R. Ueba, H. Himura, S. Masamune, S. Ohdachi, N. Mizuguchi. Rev. Sci. Instrum., 85, 033502 (2014).

[16] L. Delgado-Aparicio, J. Wallace, H. Yamazaki, P. VanMeter, L. Reusch, M. Nornberg, A. Almagari, J. Maddox, B. Luethi, M. Rissi, T. Donath, D. Den Hartog, J. Sarff, P. Weix, J. Goetz, N. Pablant, K. Hill, B. Stratton, P. Efthimion, Y. Takase, A. Ejiri. M. Rev. Sci. Instrum., 89 (10), $10 \mathrm{G116}$ (2018). DOI: 10.1063/1.5038798

[17] B. Alper, S. Dillon, A. Edwards, R. Gill, R. Robins, D. Wilson. Rev. Sci. Instrum., 68 (1), 778 (1997).

[18] E. Hollmann, L. Chousal, R. Fisher, R. Hernandez, G. Jackson, M. Lanctot, S. Pidcoe, J. Shankara, D. Taussig. Rev. Sci. Instrum., 82 (11), 113507 (2011).

[19] V. Igochine, A. Gude, M.'Maraschek, ASDEX Upgrade team. IPP report, 1/338 Mai, 2010.

[20] E. Silver, M. Bitter, K. Brau, D. Eames, A. Greenberger, K. Hill, D. Meade, W. Roney, N. Sauthoff, S. Von Goeler. Rev. Sci. Instrum., 53, 1198 (1982).

[21] L. Delgado-Aparicio, D. Stutman, K. Tritz, M. Finkenthal, R. Bell, D. Gates, R. Kaita, B. LeBlanc, R. Maingi, H. Yuh, F. Levinton, W. Heidbrink. Plasma Phys. Control. Fusion., 49 1245 (2007).

[22] B. LeBlanc, R. Bell, S. Kaye, D. Stutman, M. Bell, M. Bitter, C. Bourdelle, D. Gates, R. Maingi, S. Medley, J. Menard, D. Mueller, S. Paul, A. Roquemore, A. Rosenberg, S. Sabbagh, V. Soukhanovskii, E. Synakowski, J. Wilson and the NSTX Research Team. Nucl. Fusion, 44, 513 (2004). 
[23] B. Stratton, M. Biter, K. Hill, D. Hillis, J. Hogan, Passive Spectroscopic Diagnostics for Magnetically-Confined Fusion Plasmas. (United States: N. p., 2007). DOI: 10.2172/962715

[24] M. McGarry, P. Franz, D. Den Hartog, J. Goetz. Plasma Phys. Control. Fusion., 56, 125018 (2014).

[25] I. Furno. Fast Transient Transport Phenomena Measured by soft X-ray Emission in TCV Tokamak Plasmas. LRP 703/01, August 2001.

[26] M. Anton, H. Weisen, M. Dutch, W von der Linden, F. Buhlmann, R. Chavan, B. Marletaz, P. Marmillod, P. Paris. Plasma Phys. Controlled Fusion., 38, 1849 (1996).

[27] M. Im ıšek, J. Mlynář, V. Löffelmann, V. Weinzettl, T. Odstrčil, M. Odstrčil, M. Tomeš. Nukleonika, 61 (4), 403-8 (2016).

[28] M. Imríšek, V. Weinzettl, J. Mlynář, T. Odstrčil, M. Odstrčil, O. Ficker, J. Pinzon, C. Ehrlacher, R. Panek, M. Hron. Rev. Sci. Instrum., 85, 11E433 (2014).

[29] Podgorny M., Plasma Diagnostic Lectures (Atomizdat, M., 1968), p. 88.

[30] Zh. Qiuping, Ch. Cheng, M. Yuedong. Plasma Sci. Technol., 11 (5), 560 (2009).

[31] В.М. Тимохин, А.И. Рыкачевский, И.В. Мирошников, В.Ю. Сергеев, М.М. Кочергин, А.Н. Коваль, Е.Е. Мухин, С.Ю. Толстяков, А.В. Воронин. Письма в ЖТФ, 42 (15), 13 (2016). [V. Timokhin, A. Rykachevskii, I. Miroshnikov, V. Sergeev, M. Kochergin, A. Koval', E.E. Mukhin, S. Tolstyakov, A. Voronin. Tech. Phys. Lett., 42 (8), 775 (2016).]

[32] V. Minaev, V. Gusev, N. Sakharov, V. Varfolomeev, N. Bakharev, V. Belyakov, E. Bondarchuk, P. Brunkov, F. Chernyshev, V. Davydenko, V. Dyachenko, A. Kavin, S. Khitrov, N. Khromov, E. Kiselev, A. Konovalov, V. Kornev, G. Kurskiev, A. Labusov, A. Melnik, A. Mineev, M. Mironov, I. Miroshnikov, M. Patrov, Yu. Petrov, V. Rozhansky, A. Saveliev, I. Senichenkov, P. Shchegolev, O. Shcherbinin, I. Shikhovtsev, A. Sladkomedova, V. Solokha, V. Tanchuk, A. Telnova, V. Tokarev, S. Tolstyakov, E. Zhilin. Nucl. Fusion., 57, 066047 (2017).

[33] G. Kurskiev, S. Tolstyakov, A. Berezutskiy, V. Gusev, M. Kochergin, V. Minaev, E. Mukhin, M. Patrov, Yu. Petrov, N. Sakharov, V. Semenov, P. Chernakov. Thermonucl. Fusion., 2, 81 (2012).

[34] S. Lebedev, M. Andrejko, L. Askinazi, V. Golant, V. Kornev, L. Levin, V. Rozhansky, M. Tendler, A. Tukachinsky. Plasma Phys. Control. Fusion., 36, B289 (1994).

[35] F. Scholze, R. Klein, R. Müller. Metrologia, 43, 6 (2006).

[36] S. Luk'yanov. Hot Plasma and Controlled Nuclear Fusion (Nauka, M., 1975), p. 154.

[37] W. Karzas, R. Latter. Astrophysical J. Suppl. Ser., 6, 167 (1960).

[38] Электронный ресурс. Режим доступа: http://technoexan.ru/en/products/sildet.php

[39] P. Aruev, Yu. Kolokolnikov, N. Kovalenko, A. Legkodymov, V. Lyakh, A. Nikolenko, V. Pindyurin, V. Sukhanov, V. Zabrodsky. Nucl. Instrum. Meth. Phys. Res. A, 603, 58 (2009).

[40] П. Аруев, С. Бобашев, А. Красильщиков, А. Николаев, Д. Петров, Е. Шерстнев, Приборы и техника эксперимента, 1, 1 (2021). [P. Aruev, S. Bobashev, A. Krassilchtchikov, A. Nikolaev, D. Petrov, E. Sherstnev. Instrumen. Experimental Techniq., 64, 93 (2021). DOI: 10.1134/S0020441220060147]

[41] V. Mishin, I. Shishov, A. Minchena. Mater. Phys. Mechan., 38 (1), 40 (2018).
[42] V. Mishin, I. Shishov, O. Stolyarov, I. Kasatkin, P. Glukhov. Mater., 11, 100726 (2020).

[43] Электронный ресурс. Режим доступа: www./physics.nist.gov/PhysRefData/ASD/lines_form.html 